\title{
GOBIERNO CORPORATIVO Y REEMPLAZO DE PRESIDENTES DE LOS CONSEJOS DE ADMINISTRACIÓN EN MÉXICO
}

\author{
Martín Dávila* \\ Karen Watkins** \\ (Recibido: Julio, 2010 / Aprobado: Septiembre, 2010)
}

RESUMEN

El presente trabajo estudia la repercusión de cuatro variables de Gobierno Corporativo sobre la probabilidad de reemplazo de Presidentes de los Consejos de Administración en México. Las variables que se analizan son: vínculos familiares, vínculos empresariales, vínculos políticos y tamaño de la Junta de Directores. Las conclusiones a las que se llegan difieren, en general, del resto de la literatura revisada. Esto se atribuye a que la realidad empresarial mexicana es distinta a la observada en muchos otros países, por lo que la teoría de agencias no aplica en este caso. Palabras clave: Reemplazos, Gobierno Corporativo, Junta Directiva, teoría de agencias

Clasificación JEL: G34

\begin{abstract}
This paper studies the effects of four Corporate Governance variables over the turnover probability of Mexican Board of Director's Presidents. The variables

* Facultad de Economía, Universidad Popular Autónoma del Estado de Puebla, México. Correo electrónico: <martin.davila@upaep.mx>.

* Facultad de Economía y Centro de Investigación e Inteligencia Económica, Universidad Popular Autónoma del Estado de Puebla. Correo electrónico: <karen.watkins.f@gmail.com>.

Agradecemos a Alfonso Mendoza, Ignacio Ibarra y Mariano Rojas por sus valiosos comentarios.
\end{abstract}


analyzed are: family links, business groups, political links, and size of the Board of Directors. The conclusions reached in general differ from the rest of the literature reviewed. This is explained by the fact that the Mexican entrepreneurial reality is different from many other countries; therefore, agency theory does not apply in this case.

Keywords: Turnovers, Corporate Governance, Board of Directors, agency theory JEL Classification: G34

\section{INTRODUCCIÓN}

El desempeño de las empresas está relacionado con la gestión de los Presidentes de los Consejos de Administración. El reemplazo de estos funcionarios representa una decisión importante por parte de los accionistas de las firmas, por sus repercusiones sobre el rendimiento empresarial. Es también una medida de Gobierno Corporativo que favorece la alineación de objetivos empresariales ya que, la amenaza creíble de un reemplazo limita a los agentes de los beneficios privados obtenidos mediante el poder en las empresas.

Existen diferentes motivos por los que se llegan a dar estos reemplazos. Las variaciones del mercado representan uno de estos factores puesto que, no todos los Presidentes de los Consejos de Administración son aptos para cualquier contingencia. Un segundo factor se refiere a la competencia en el mercado de directivos; entre más competitivo sea este mercado mayor es la probabilidad de que ocurran los reemplazos. El pobre desempeño empresarial representa una tercera causa para cambiar a los directores, con lo que se espera un mejoramiento en los resultados corporativos. Existen también ciertos mecanismos de Gobierno Corporativo que se relacionan con la probabilidad de reemplazo del Presidente de la Junta Directiva.

El presente trabajo estudia la repercusión de cuatro variables de Gobierno Corporativo sobre la probabilidad de reemplazo de los Presidentes de los Consejos de Administración en México. Las variables que se analizan son: vínculos familiares, vínculos empresariales, vínculos políticos y el tamaño de la Junta de Directores. Adicionalmente, se busca establecer la relación entre el desempeño y el tamaño de 
Gobierno corporativo y ReEmplazo de Presidentes de

los Consejos de Administración en México

la firma con el cambio de estos altos funcionarios. Finalmente, se contemplan las posibles diferencias sobre las decisiones de reemplazo de estos directivos durante situaciones de crisis financiera y posteriores a ésta, así como su impacto sobre los resultados corporativos.

Las conclusiones a las que se llegan difieren, en general, del resto de la literatura revisada. Esto se debe a que la realidad empresarial mexicana es distinta a la observada en muchos otros países. La alta incidencia de dueños mayoritarios dentro de la presidencia de las Juntas Directivas hace que las variables de Gobierno Corporativo estudiadas no tengan impacto en las decisiones de reemplazo. Incluso la significancia de variables tales como el tamaño de la firma y el desempeño empresarial sobre la probabilidad de reemplazo se explica con argumentos distintos, ajenos a la teoría de agencias.

Este trabajo está organizado de la siguiente manera: la sección dos presenta la revisión de la literatura y la teoría relevante sobre las variables de Gobierno Corporativo y sus implicaciones en la probabilidad de reemplazo de los altos funcionarios. Este apartado termina con las consecuencias del reemplazo sobre el desempeño empresarial. La sección tres presenta los objetivos, hipótesis, los datos y las variables empleadas, así como la metodología del estudio. Para verificar las hipótesis se utilizan modelos Logit. En la sección cuatro se presenta la estadística descriptiva pertinente para esta investigación. La sección cinco hace referencia a los resultados econométricos y finalmente la sección seis concluye.

\section{REVISIÓN DE LA LITERATURA}

El reemplazo tanto del Director General como del Presidente del Consejo de Administración de cualquier compañía se puede dar por diferentes motivos. ${ }^{1}$ Uno de

\footnotetext{
1 Varios autores, entre los que se encuentra Florou (2005), muestran que existe una relación directa entre el reemplazo del Director General y del Presidente de la Junta de Directores. Cuando se da uno, repercute en el abandono de funciones del otro debido a la gran proximidad que tienen dentro de una empresa.
} 
ellos se refiere a las variaciones que se presentan en el mercado; esto es, existe la posibilidad de que el funcionario ya no tenga la capacidad para continuar dirigiendo el destino de la compañía debido al tamaño o complejidad que ha alcanzado el mercado al que se está enfrentando (ver Hermalin y Weisbach, 1998; Fee y Hadlock, 2000; Fung y Rui, 2006 y Jenter y Kanaan, 2006). Otro causante del reemplazo es el mercado laboral de ejecutivos y directivos, el cual implica tanto competencia interna (entre funcionarios dentro de la misma firma) como externa (ver Fama, 1980; McNeil et al., 2004 y Nair, 2005; Manne, 1965 y Fama y Jensen, 1983a). Una tercera motivación para sustituir a un alto ejecutivo o directivo es el bajo desempeño empresarial. Los accionistas de empresas que experimentan rendimientos pobres vigilan más de cerca a sus administradores y directores, lo cual favorece el reemplazo (ver Jovanovic, 1979; Coughlan y Schmidt, 1985; McKenna, 1986; Murphy, 1986; Warner et al., 1988; Weisbach, 1988; Conyon y Florou, 2002; Fee y Hadlock, 2004 y Jenter y Kanaan, 2006).

La posibilidad de sustituir agentes, tales como el Director General o el Presidente del Consejo de Administración de una compañía, favorece la alineación de objetivos empresariales. La Porta et al. (1998); Nenova (2000); Claessens et al. (2002) y Dyck y Zingales (2004) sostienen que el sistema legal protege a los inversionistas mediante el otorgamiento de derechos para disciplinar a los administradores por medio de su reemplazo. Lo anterior puede ser visto como una forma para hacer cumplir los contratos tal y como están diseñados, de manera que limiten a los gerentes de los beneficios privados obtenidos mediante el control de las firmas. En este sentido, los reemplazos constituyen un mecanismo de Gobierno Corporativo (ver Warner et al., 1988; Harrison et al., 1988; Manne, 1965 y Gibson, 2003). Por otro lado, existen otras medidas de Gobierno Corporativo que a su vez se relacionan con los reemplazos.

\subsection{MECANISMOS DE GOBIERNO CORPORATIVO Y SU RELACIÓN CON LOS REEMPLAZOS}

Ciertos mecanismos de Gobierno Corporativo implementados por las empresas

pueden influenciar la probabilidad de que se lleguen a presentar reemplazos de 
Gobierno corporativo y ReEmplazo de Presidentes de

los Consejos de Administración en MéXico

los altos ejecutivos o directores. Algunas de estas variables son: la existencia de vínculos familiares, los vínculos empresariales, los vínculos políticos y el tamaño de la Junta de Directores. ${ }^{2}$

\subsubsection{VÍNCULOS FAMILIARES Y REEMPLAZOS DE ALTOS FUNCIONARIOS}

Los gerentes-dueños (o familias) que dirigen empresas tienen motivaciones personales y financieras para incrementar el valor de sus compañías. Pero al mismo tiempo, también tienen fuertes incentivos para obtener beneficios personales y control sobre las firmas, a expensas de los accionistas minoritarios y demás interesados $^{3}$ (ver Anderson y Reeb, 2003; Villalonga y Amit, 2006 y Bennedsen et al., 2007). Según Hillier y McColgan (2004), la mayoría de estas compañías contratan a miembros de sus familias como ejecutivos y directores de las mismas, por lo que el nepotismo es una característica importante en este tipo de organizaciones. De acuerdo a Lausten (2002) y Volpin (2002), bajo estas condiciones la relación entre el reemplazo y el desempeño de la firma es débil, lo cual evidencia el atrincheramiento (ver Denis et al., 1997; Dahya et al., 1998 y Hillier y McColgan, 2004). Esto lo ejemplifican Tosi et al. (2003) al mostrar que los Directores Generales, que no son miembros de la familia, tienen mayor probabilidad de reemplazo que los ejecutivos que sí forman parte de la misma. Es así que el vínculo familiar en una firma hace que la probabilidad de que cambie un ejecutivo o director se reduzca.

Por el contrario, Kaplan (1994) y Firth et al. (2006) argumentan que los accionistas de empresas familiares supervisan minuciosamente a sus ejecutivos y directores (aún si éstos son miembros de la familia), ya que son muchos sus intereses en la firma. Es así que, la probabilidad de que estos agentes sean despedidos es mayor que en empresas no familiares, particularmente si se presenta un bajo desempeño empresarial.

\footnotetext{
2 Para una exposición sobre mecanismos de Gobierno Corporativo en México ver Dávila y Watkins (2008).

${ }^{3}$ Que es congruente con la hipótesis de atrincheramiento expuesta por Fama y Jensen (1983a).
} 
Martín DÁvila / Karen WatKins

\subsubsection{VÍNCULOS EMPRESARIALES Y REEMPLAZOS DE ALTOS FUNCIONARIOS}

El vínculo empresarial implica cierto grado de integración entre compañías. ${ }^{4}$ Éste permite que exista un mercado interno de ejecutivos y miembros de los Consejos de Administración, los cuales compiten por dirigir los destinos de las empresas más importantes del grupo. Aquellos que logran participar en las firmas que forman parte de la cúspide de la pirámide empresarial obtienen mayor prestigio y poder, lo cual constituye un objetivo de los agentes.

Chang y Shin (2006) encuentran que la probabilidad de que se presenten reemplazos de altos funcionarios es mayor en aquellas compañías que forman parte de un grupo empresarial. El mercado interno de ejecutivos y miembros de los Consejos de Administración reduce el problema de la información asimétrica en la contratación, haciendo que estos agentes sean más fácilmente promovidos y destituidos.

Por el contrario, autores tales como Gompers et al. (2003), Fich y Shivdasani (2006), Bebchuk et al. (2004, 2008), Bhagat y Bolton (2008) y Maury (2006) señalan que el atrincheramiento está también presente en este tipo de compañías. La probabilidad de destituir a un alto funcionario está negativamente asociada con la cuota de poder que tiene el mismo en el grupo empresarial. Es por ello que la probabilidad de reemplazar a un ejecutivo o director puede ser menor que en firmas sin este tipo de vínculos.

\subsubsection{VÍNCULOS POLÍTICOS Y REEMPLAZOS DE ALTOS FUNCIONARIOS}

De acuerdo con Faccio (2006a), el vínculo político se establece cuando algún miembro de la burocracia gubernamental forma parte de la empresa. Esto es, pertenece a la Junta Directiva (Consejo de Administración), es un ejecutivo o un accionista de la firma.

El político-empresario tiene menor probabilidad de ser reemplazado, cuando su presencia implica mejores posibilidades de ganar licitaciones y mayor acceso 
Gobierno corporativo y ReEmplazo de Presidentes de

los Consejos de Administración en México

a ciertos mercados financieros (ver Agrawal y Knoeber, 2001 y Fan et al., 2008). Sin embargo, estos agentes muchas veces carecen de experiencia empresarial, lo cual los vuelve más vulnerables ante el reemplazo. Esta situación se agudiza cuando la firma muestra resultados desfavorables (ver Roberts,1990; Fisman, 2001; Mak y Li, 2001 y Sung y Tong, 2003).

\subsubsection{TAMAÑO DE LAS JUNTAS DIRECTIVAS Y REEMPLAZOS DE ALTOS FUNCIONARIOS}

Para Mak y Li (2001) y Ferris et al. (2003), las Juntas Directivas (Consejos de Administración) pequeñas poseen mayores problemas de información asimétrica que las grandes; por ende, pueden cometer más errores en las decisiones de contratación de los altos ejecutivos y directores. Esto repercute posteriormente en la substitución de funcionarios, por lo que la probabilidad de reemplazo puede ser superior que en empresas con Juntas Directivas Grandes. Acorde con esto, Lipton y Lorsch (1992); Jensen (1993); Yermack (1996) y Eisenberg et al. (1998) sostienen que las Juntas Directivas pequeñas (entre ocho y diez miembros) gozan de mejor coordinación y eficiencia en las actividades de monitoreo de las firmas. Por lo tanto, la probabilidad de reemplazar agentes puede ser mayor que en aquellas compañías con Juntas Directivas más grandes.

Sin embargo, autores como Bhagat y Black (2002) no encuentran una relación entre el tamaño de la Junta de Directores y la probabilidad de reemplazo de altos funcionarios.

\subsection{OTRAS VARIABLES QUE INFLUYEN EN EL REEMPLAZO DE ALTOS FUNCIONARIOS}

Las variables de Gobierno Corporativo antes expuestas se relacionan con la posibilidad de sustituir ejecutivos y directores. Asimismo, existen otros factores que influyen en la probabilidad de reemplazar funcionarios. La literatura revisada menciona el tamaño y el desempeño de la firma como variables relevantes, las cuales se describen a continuación. 
Martín DÁvila / Karen WatKins

\subsubsection{TAMAÑO DE LAS FIRMAS Y REEMPLAZOS DE ALTOS FUNCIONARIOS}

El tamaño de la empresa es una variable importante que hay que considerar al estudiar los reemplazos de los funcionarios. Según Lausten (2002) y Conyon (1998), entre más grande sea una firma más baja es la probabilidad de que se despida a un alto ejecutivo (lo mismo aplica para los miembros del Consejo de Administración). Esto se debe al conocimiento que este agente ha adquirido en el manejo complejo de una organización de magnas dimensiones, lo cual es difícil de sustituir. Además, entre más grande sea una firma más fácil resulta esconder errores de gestión, lo cual evita que el funcionario sea fácilmente despojado de su cargo (ver Agrawal y Knoeber,1996).

Adicionalmente a lo anterior, en las empresas grandes el salario y las compensaciones que reciben los altos funcionarios tienden a ser mayores que en las demás corporaciones (ver Perry, 1999). Esto permite contratar personas más capacitadas y eficientes disminuyendo las probabilidades de despido. Las favorables condiciones salariales ayudan también a aliviar el problema de agencias, alineando los intereses de los accionistas y los administradores (tal y como lo mencionan Aggarwal y Samwick, 1999; Bebchuk et al., 2002 y Bebchuk y Fried, 2003); lo anterior reduce la probabilidad de reemplazo.

En contraste Denis et al. (1997) y Warner et al. (1988) señalan que, en las empresas de gran tamaño la probabilidad de sustituir altos funcionarios es mayor que en las demás. Estos autores argumentan que, en este tipo de empresas existe mayor propensión a despedir agentes ante caídas en el desempeño empresarial; además, la competencia por puestos en el mercado laboral interno de las empresas grandes es superior que en el resto, lo cual favorece los reemplazos.

\subsubsection{DESEMPEÑO DE LAS EMPRESAS Y REEMPLAZOS DE ALTOS FUNCIONARIOS}

De acuerdo a la teoría de agencias, el agente muchas veces trabaja en concordancia con los objetivos de los accionistas por temor a ser despedido de su cargo. 
Gobierno corporativo y ReEmplazo de Presidentes de

los Consejos de Administración en México

El objetivo principal de una empresa es maximizar las utilidades; esto significa que la probabilidad de que un alto funcionario sea sustituido aumenta cuando la empresa presenta bajo desempeño (ver Conyon, 1998; Alchian y Demsetz, 1972; Hillier et al., 2005; Fama y Jensen, 1983b; Hoskisson et al. 1999; Lausten, 2002; Maury, 2006 y Fan et al., 2007). Los inversionistas generalmente ven con buenos ojos los anuncios de cambios de los agentes debido a que éstos implican un mejoramiento en los resultados financieros de la firma (ver Barro y Barro, 1990; Jensen y Murphy, 1990; Gibbons y Murphy, 1990; Denis y Denis, 1995; Cosh y Hughes, 1997; Parrino, 1997; DeFond y Park, 1999; Kaplan, 1994; Coffee, 1999; Weisbach, 1988; Bonnier y Bruner, 1989 y Huson et al., 2004). No obstante, Khanna y Poulsen (1995) y Firth et al. (2006) encuentran evidencia empírica al señalar que, una vez que se da el reemplazo de un alto ejecutivo el desempeño de la empresa cae. Esto se puede explicar por los costos de aprendizaje, por las decisiones incorrectas de contratación o por lineamientos de gestión inadecuados.

\subsection{DIFERENCIAS EN DECISIONES DE REEMPLAZO DURANTE SITUACIONES DE CRISIS Y NORMALES}

Chang y Shin (2006) y Heaney et al. (2007) mencionan que es mayor la probabilidad de que se dé el reemplazo de un alto ejecutivo posterior a una crisis, especialmente cuando la firma presenta bajo desempeño empresarial. Esto se debe a las políticas más exigentes de monitoreo implementadas por las empresas como consecuencia de la crisis sistémica.

En tiempos de crisis no se espera una alta correlación entre los resultados empresariales y los reemplazos. Bajo estas circunstancias, no es posible culpar a los agentes de la disminución del desempeño empresarial, ${ }^{5}$ ya que la crisis afecta a todo el mercado y es exógena a la firma; puede atribuirse a factores macroeconómicos locales o internacionales, problemas políticos, falta de competitividad $u$ otras situaciones que se escapan del control de la empresa.

\footnotetext{
5 A menos que se cumpla la teoría del "chivo expiatorio".
} 
Martín DÁvila / Karen WatKins

\section{OBJETIVOS, HIPÓTESIS, DATOS, VARIABLES Y METODOLOGÍA}

\subsection{OBJETIVOS E HIPÓTESIS}

El objetivo principal que se persigue en esta investigación es, encontrar factores de Gobierno Corporativo que influyan en la probabilidad de reemplazo de los Presidentes de los Consejos de Administración (Juntas Directivas) en México. Se busca también establecer la relación entre el desempeño y el tamaño de la firma con el cambio de estos altos funcionarios. Para esto se utiliza información de las empresas listadas en la Bolsa Mexicana de Valores (BMV) del año 1990 al 2000.

Por otro lado, se estudian las posibles diferencias sobre las decisiones de reemplazo de los Presidentes de las Juntas Directivas durante situaciones normales y de crisis. Específicamente, se comparan los períodos antes de la crisis financiera de 1994 (1990-1993) con los tiempos de crisis (1994-1995) y la época posterior a la misma (1996-2000).

De acuerdo con lo anterior, se plantean las siguientes hipótesis:

H1: Los vínculos familiares se relacionan de forma inversa con la probabilidad de reemplazo de los Presidentes de las Juntas Directivas. Autores como Stulz (1988), Fama y Jensen (1983a) y Hillier y McColgan (2004) señalan que la mayoría de estas empresas contratan a miembros de sus familias como altos funcionarios de las mismas, lo cual propicia mayor estabilidad laboral.

H2: Los vínculos empresariales favorecen la probabilidad de que un Presidente del Consejo de Administración sea reemplazad. Gompers et al. (2003); Bebchuk et al. (2004) y Bhagat y Bolton (2008) mencionan que los directivos de este tipo de firmas tienen altos grados de poder, por lo que sustituirlos es menos probable que en otro tipo de organizaciones.

H3: El vínculo político fomenta que los Presidentes de las Juntas Directivas tengan una menor probabilidad de ser cambiados. Faccio et al. (2006b) argumentan que, los políticos-empresarios se afianzan de sus cargos debido a las concesiones y negociaciones que logran obtener con el gobierno (las cuales benefician a la compañía que representan). 
Gobierno corporativo y ReEmplazo de Presidentes de

los Consejos de Administración en MéXico

H4: Entre más grande sea el tamaño de la Junta Directiva, menor es la probabilidad de reemplazo para el Presidente del Consejo de Administración. Autores como Mak y Li (2001) y Ferris et al. (2003) muestran que las firmas con Juntas Directivas Grandes tienen mayor capacidad de contratar acertadamente altos ejecutivos y directores, lo cual favorece la estabilidad de sus puestos.

Existen otras variables que pueden influir en la remoción de los Presidentes de las Juntas Directivas. Dos de ellas vastamente mencionadas en la literatura son el tamaño y el desempeño de las firmas. Son de esperar las siguientes relaciones:

H5: La probabilidad de reemplazo del Presidente del Consejo de Administración se relaciona inversamente con el tamaño de la empresa. Según Lausten (2002), entre más grande sea una firma, más compleja es su gestión y, por ende, es más baja la probabilidad de que se sustituya a un alto funcionario.

H6: Un bajo desempeño empresarial implica una mayor probabilidad de reemplazo del Presidente de la Junta Directiva. Barro y Barro (1990); Jensen y Murphy (1990); Gibbons y Murphy (1990); Denis y Denis (1995); Cosh y Hughes (1997); Parrino (1997); DeFond y Park (1999); Kaplan (1994); Coffee (1999) y Huson et al. (2004) presentan evidencia de que los directivos son forzados a dejar las firmas cuando hay una larga y significativa caída en los resultados empresariales.

Respecto a las diferencias sobre las decisiones de reemplazo de los Presidentes de las Juntas Directivas, durante situaciones normales y de crisis, se esperan los siguientes resultados:

H7: El número de reemplazos de los Presidentes de los Consejos de Administración se incrementa después de una crisis. De acuerdo a Chang y Shin (2006) y Heaney et al. (2007), esto se debe al minucioso monitoreo y control implementados en las empresas como consecuencia de la crisis. 
H8: En épocas de crisis cae el número de reemplazos de los Presidentes de la Juntas Directivas. Durante las crisis no es posible responsabilizar a estos agentes por la caída de los resultados de las firmas ya que, este episodio afecta negativamente a todo el mercado.

Por último, se espera la siguiente relación entre el reemplazo del Presidente del Consejo de Administración y el desempeño empresarial:

H9: De acuerdo con autores tales como Barro y Barro, 1990; Jensen y Murphy, 1990 y Gibbons y Murphy, 1990, después del reemplazo se presenta un mejoramiento en el desempeño de la firma.

\subsection{DATOS}

La información recopilada es de 171 firmas que cotizan (o cotizaron) en la Bolsa Mexicana de Valores (BMV) de 1990 a 2000. Todas las variables monetarias son presentadas en términos reales, a pesos del año 2000.

Para los primeros años del período de tiempo que maneja la investigación, se obtienen los datos financieros (rendimiento sobre los activos como variable de desempeño y activos totales como variable de tamaño empresarial) directamente de la BMV. Esta información se encuentra disponible a manera de microfilmación. La gran mayoría de los datos es obtenida de manera electrónica en el Sistema Integral de Valores Automatizados (SIVA, por sus siglas en castellano), así como en Infosel Financiero.

La información sobre los miembros de las Juntas Directivas se registra de manera manual del Anuario Financiero que se encuentra en el Centro de Información de la BMV. Los nombres de los políticos-empresarios se obtienen directamente de la Oficina de la Presidencia de la República y del Congreso de la Nación y sus respectivas páginas en internet. A partir de esta información se construyen las variables categóricas de Gobierno Corporativo: vínculo familiar, vínculo empresarial, vínculo político y tamaño de la Junta Directiva. 
Gobierno corporativo y ReEmplazo de Presidentes de

los Consejos de Administración en México

\subsection{VARIABLES}

Se toma como variable dependiente el reemplazo de los Presidentes de los Consejos de Administración (Juntas Directivas) en México. ${ }^{6}$ Se entiende por reemplazo el cambio del Presidente de la Junta Directiva de una firma en un año en particular. Esta variable toma el valor de uno (1) en el año en que se presenta el reemplazo y cero $(0)$ en los demás casos. ${ }^{7}$

Las variables de Gobierno Corporativo que se contemplan son:

1. Vínculo familiar (directores-dueños) (Familiar): Es una variable dicotómica (dummy) que toma el valor de uno cuando en un año en particular el Presidente de la Junta Directiva comparte al menos un apellido con otro(s) miembro(s) del mismo Consejo de Administración. Es cero en los demás casos.

2. Vínculo empresarial (Empresarial): Variable dicotómica (dummy) que es cero cuando el Presidente de la Junta Directiva de una empresa no forma (o ha formado) parte de otra Junta Directiva en un mismo año durante el período 1990-2000. Toma el valor de uno cuando el Presidente de la Junta Directiva es (o ha sido) miembro de otra u otras Juntas Directivas, para el mismo año, en el período comprendido entre 1990-2000.

3. Vínculo político (Político): Variable dicotómica (dummy) que es cero cuando el Presidente del Consejo de Administración no es (o no ha sido) parte de la burocracia gubernamental. Toma el valor de uno cuando el Presidente de la Junta de Directores es (o ha sido) Gobernador de algún Estado de la República mexicana, Diputado Federal electo, miembro del Senado o Secretario de Estado (en el espacio de tiempo comprendido entre 1990-2000).

4. Tamaño de la Junta de Directores (TJD): Representa una variable dicotómica (dummy) para el tamaño de las Juntas Directivas: uno para Juntas Directivas Grandes (mayores o iguales a 19 miembros) y cero para Juntas Directivas pe-

${ }^{6}$ En México, la información histórica de los altos ejecutivos no es pública; por ende, se trabaja exclusivamente con los Presidentes de los Consejos de Administración.

${ }^{7}$ De 1990-2000, una firma puede presentar más de un reemplazo. Para estas empresas, la variable Reemplazo toma el valor de uno más de una vez. 
queñas (menores a 19 miembros). De los anuarios financieros se capturaron tanto los nombres de los miembros de las Juntas Directivas, como el número de miembros de las mismas. El número de observaciones fue de $1234 \mathrm{y}$ se obtuvo un promedio para todas las empresas de 19.31 miembros. El máximo de miembros en una Junta Directiva fue de 60 y el valor mínimo para una Junta de Directores fue de 4. La desviación estándar fue de 7.58 miembros. Con esta información se tomó la decisión de que el punto de inflexión fuera de 19 miembros para las Juntas Directivas Grandes.

Las variables de control empleadas son:

1. Cambio en el desempeño de la empresa $\left(\Delta \mathrm{ROA}_{\mathrm{t}-2}\right)$ : Se refiere al cambio en el rendimiento sobre los activos, rezagado dos períodos. Éste permite observar cómo las variaciones en el desempeño pasado influyen en la probabilidad de reemplazo de los Presidentes de las Juntas Directivas. $\triangle$ ROA se construye como $\mathrm{ROA}_{t}-\mathrm{ROA}_{\mathrm{t}-1}$.

2. Tamaño de la empresa (Tamaño): El tamaño de la empresa está representado por el logaritmo natural de los activos totales.

\subsection{METODOLOGÍA}

Para estimar la probabilidad de reemplazo de los Presidentes de los Consejos de Administración, de acuerdo a las características de Gobierno Corporativo de las firmas, se utiliza un modelo Logit. La función estimada es la siguiente:

$$
\begin{array}{ll}
\text { Reemplazo }=\mathrm{f} & {\left[\text { Familiar }_{t-1}, \text { Empresarial }_{\mathrm{t-1}-1}, \text { Político }_{\mathrm{t}-1}, \mathrm{TJD},\right. \text { Tamaño, }} \\
& \left.\Delta \mathrm{ROA}_{\mathrm{t}-2} *(\text { Crisis }), \Delta \mathrm{ROA}_{\mathrm{t}-2} *(\text { Post-Crisis })\right]
\end{array}
$$

Las variables vínculo familiar, vínculo empresarial y vínculo político se construyen a partir de la figura del Presidente de la Junta Directiva; es decir, si éste es reemplazado, es probable que en ese mismo año también cambie el valor de la variable de Gobierno Corporativo en cuestión. Por ende, para evitar errores de especificación, en (1) estas tres variables se rezagan un período. 
Gobierno corporativo y ReEmplazo de Presidentes de

los Consejos de Administración en MéXico

Por otro lado, en (1) se hace la interacción del cambio en el rendimiento sobre los activos (rezagado dos períodos) con los años de crisis y de post-crisis. Esto

permite diferenciar el efecto de $\Delta \mathrm{ROA}_{\mathrm{t}-2}$ sobre la probabilidad de reemplazo del Presidente de la Junta Directiva en la época de crisis y posterior a la misma.

\section{ESTADÍSTICA DESCRIPTIVA}

\subsection{REEMPLAZOS Y CARACTERÍSTICAS DE GOBIERNO CORPORATIVO}

En la Tabla 1 se muestran los reemplazos que se dieron en las empresas mexicanas, de acuerdo a sus características de Gobierno Corporativo. La muestra consta de un total de 171 firmas no-financieras, listadas en la Bolsa Mexicana de Valores. Se puede observar que, de esa muestra, en total se presentaron reemplazos en sólo 121 ocasiones. Para el período de pre-crisis (1990-1993) únicamente se tuvieron 31, mismos que empezaron a aumentar ligeramente a través del tiempo. Para el período de crisis (1994-1995) fueron 40 y en el período de post-crisis (1996-2000) se llegaron a 50 reemplazos.

Entonces, con referencia a la Tabla 1, entenderemos por reemplazo al cambio del Presidente del Consejo de Administración de una empresa, en un año en particular. Es así que, para una misma firma puede haber más de un reemplazo en el período de 1990-2000. Las 4 características (eliminando la categoría "todas las firmas") que se muestran en la tabla no son mutuamente excluyentes. Es decir, cada empresa puede pertenecer a más de una categoría de Gobierno Corporativo. El vínculo familiar se establece cuando el Presidente de la Junta Directiva de una empresa tiene en un año en particular, algún apellido igual a otro miembro del mismo Consejo de Administración. Se considera que una firma tiene vínculo familiar en la pre-crisis, crisis y/o post-crisis si posee este nexo en al menos uno de los años del período en cuestión. Se tiene vínculo empresarial cuando el Presidente de una Junta Directiva está participando en el mismo año como miembro de otra u otras Juntas Directivas. Se cataloga a una empresa con vínculo empresarial en la pre-crisis, crisis y/o post-crisis si tiene este vínculo en al menos uno de los 
años del período en cuestión. Cuando el Presidente de la Junta de Directores de una compañía ha sido Gobernador de algún Estado, Diputado Federal electo mediante votación popular, Senador de la República o Secretario de Estado (de 1990 a 2000), se dice que esa empresa posee vínculo político. Se contempla que una firma tiene vínculo político en la pre-crisis, crisis y/o post-crisis si posee este nexo en al menos uno de los años del período en cuestión. Se considera una empresa con Junta de Directores Grande cuando el número de miembros es de 19 o más. Si la empresa en particular tiene Junta Directiva Grande en al menos 60\% de los años del período en estudio (pre-crisis, crisis o post-crisis), entonces se contabiliza como una empresa con Junta Directiva Grande durante ese tiempo.

TABLA 1

Número de reemplazos de Presidentes de las Juntas Directivas en México durante las épocas de pre-crisis (1990-1993), crisis (1994-1995) y post-crisis (1996-2000), de acuerdo a distintas características de Gobierno Corporativo

\begin{tabular}{|c|c|c|c|c|c|c|c|}
\hline \multirow{3}{*}{$\begin{array}{l}\text { Características de las firmas } \\
\text { Todas las firmas }\end{array}$} & \multirow{2}{*}{\multicolumn{2}{|c|}{$\begin{array}{l}\text { Todos los } \\
\text { años }\end{array}$}} & \multicolumn{4}{|c|}{ Número de Reemplazos } & \multirow[b]{2}{*}{ Post-Crisis } \\
\hline & & & \multicolumn{2}{|c|}{ Pre-Crisis } & \multicolumn{2}{|c|}{ Crisis } & \\
\hline & 121 & $100 \%$ & 31 & $100 \%$ & 40 & $100 \%$ & $50100 \%$ \\
\hline Vínculo familiar & 58 & $48 \%$ & 18 & $58 \%$ & 16 & $40 \%$ & $24 \quad 48 \%$ \\
\hline Vínculo empresarial & 61 & $50 \%$ & 15 & $48 \%$ & 28 & $70 \%$ & $1836 \%$ \\
\hline Vínculo político & 25 & $21 \%$ & 3 & $10 \%$ & 11 & $28 \%$ & $1122 \%$ \\
\hline Junta Directiva Grande & 47 & $39 \%$ & 14 & $45 \%$ & 15 & $38 \%$ & $18 \quad 36 \%$ \\
\hline
\end{tabular}

Fuente: Elaboración propia.

Se observa que existe una relación entre los cambios de los Presidentes de las Juntas Directivas y los vínculos familiares y empresariales pues, las empresas con estas características son los que más reemplazos presentan. El vínculo familiar generó la cantidad más alta de reemplazos en la pre-crisis y la post-crisis, mientras que el vínculo empresarial lo tuvo en la crisis. De acuerdo a la literatura, los dueños de las empresas familiares realizan un mayor monitoreo sobre sus ejecutivos y 
Gobierno corporativo y ReEmplazo de Presidentes de

los Consejos de Administración en MéXico

directores (aún si éstos son miembros de la familia), ya que tienen más intereses en juego (ver Kaplan, 1994 y Firth et al., 2006). Es por eso que la posibilidad de que los directivos sean destituidos aumenta en este tipo de empresas, sobre todo si existe un desempeño empresarial pobre. En cuanto a la asociación entre el reemplazo y el vínculo empresarial, se observa que en estas estructuras de grupo existe un mercado interno de ejecutivos y miembros de los consejos de administración, lo cual favorece la posibilidad del reemplazo. Los problemas de información asimétrica se reducen, sobre todo en el momento de las contrataciones, por lo que los agentes son más fáciles de ser promovidos e incluso removidos de sus cargos (ver Chang y Shin, 2006).

\subsection{ESTRATEGIAS DE GOBIERNO CORPORATIVO Y REEMPLAZOS}

En la Tabla 2 se muestra la repercusión de las decisiones o "estrategias" de Gobierno Corporativo seguidas por las empresas mexicanas, durante los tres subperíodos de estudio, sobre los reemplazos de los Presidentes de los Consejos de Administración de las empresas mexicanas consideradas. Estas estrategias son combinaciones de las variables de Gobierno Corporativo estudiadas.

Con respecto a todo el período de estudio (1990-2000), la mayor cantidad de reemplazos (34) se tuvieron en aquellas empresas que no presentaron ninguna de las estrategias de Gobierno Corporativo consideradas (NGC), lo cual hace sospechar que estas variables no son las más relevantes en las decisiones de reemplazo en México (lo cual no se observa en la literatura para otros países). A esto le sigue una estrategia en la que se combinan el vínculo familiar, empresarial y las Juntas Directivas Grandes (FEG-15); de ahí en adelante, se tienen reemplazos en las empresas que sólo presentaron vínculos empresariales (12) y familiares (11), así como en aquellas que combinaron los vínculos familiares, empresariales, políticos y Juntas Directivas Grandes (FEPG-10); estas son las cinco estrategias dominantes.

Para la Tabla 2, entenderemos por reemplazo al cambio del Presidente del Consejo de Administración de una empresa en un año en particular. Es así que, para una misma firma puede haber más de un reemplazo en el período de 1990-2000. Las 16 características (excluyendo la categoría "todas las firmas") que se muestran son

mutuamente excluyentes y exhaustivas. El vínculo familiar se establece cuando 


\section{TABLA 2}

Número de reemplazos de Presidentes de las Juntas Directivas en México durante las épocas de pre-crisis (1990-1993), crisis (1994-1995) y post-crisis (1996-2000), de acuerdo a distintas estraegias de Gobierno Corporativo

\begin{tabular}{|c|c|c|c|c|c|c|c|c|}
\hline \multirow[b]{2}{*}{ Características de las firmas } & \multicolumn{8}{|c|}{$\underline{\text { Número de Reemplazos }}$} \\
\hline & \multicolumn{2}{|c|}{ Todos los años } & \multicolumn{2}{|c|}{ Pre-Crisis } & \multicolumn{2}{|c|}{ Crisis } & \multicolumn{2}{|c|}{ Post-Crisis } \\
\hline Todas las firmas & 121 & $100 \%$ & 31 & $100 \%$ & 40 & $100 \%$ & 50 & $100 \%$ \\
\hline Vínculo familiar & 11 & $9 \%$ & 4 & $13 \%$ & 2 & $5 \%$ & 5 & $10 \%$ \\
\hline Vínculo empresarial & 12 & $10 \%$ & 4 & $13 \%$ & 7 & $17 \%$ & 1 & $2 \%$ \\
\hline Vínculo político & 1 & $1 \%$ & 0 & $0 \%$ & 0 & $0 \%$ & 1 & $2 \%$ \\
\hline Junta Directiva Grande (JDG) & 2 & $2 \%$ & 1 & $3 \%$ & 0 & $0 \%$ & 1 & $2 \%$ \\
\hline Vínculo familiar y empresarial & 9 & $7 \%$ & 3 & $10 \%$ & 3 & $8 \%$ & 3 & $6 \%$ \\
\hline Vínculo familiar y político & 1 & $1 \%$ & 0 & $0 \%$ & 0 & $0 \%$ & 1 & $2 \%$ \\
\hline Vínculo familiar y JDG & 8 & $7 \%$ & 3 & $10 \%$ & 0 & $0 \%$ & 5 & $10 \%$ \\
\hline Vínculo empresarial y político & 3 & $2 \%$ & 0 & $0 \%$ & 1 & $2 \%$ & 2 & $4 \%$ \\
\hline Vínculo empresarial y JDG & 5 & $4 \%$ & 1 & $3 \%$ & 3 & $8 \%$ & 1 & $2 \%$ \\
\hline Vínculo político y JDG & 2 & $2 \%$ & 1 & $3 \%$ & 0 & $0 \%$ & 1 & $2 \%$ \\
\hline Vínculo familiar, empresarial y político & 3 & $2 \%$ & 0 & $0 \%$ & 2 & $5 \%$ & 1 & $2 \%$ \\
\hline Vínculo familiar, empresarial y JDG & 15 & $13 \%$ & 7 & $23 \%$ & 4 & $10 \%$ & 5 & $10 \%$ \\
\hline Vínculo familiar, político y JDG & 1 & $1 \%$ & 1 & $3 \%$ & 0 & $0 \%$ & 0 & $0 \%$ \\
\hline Vínculo empresarial, político y JDG & 4 & $3 \%$ & 0 & $0 \%$ & 3 & $8 \%$ & 1 & $2 \%$ \\
\hline Vínculo familiar, empresarial, político y JDG & 10 & $8 \%$ & 1 & $3 \%$ & 5 & $12 \%$ & 4 & $8 \%$ \\
\hline Firmas con ninguna de estas características & 34 & $28 \%$ & 5 & $16 \%$ & 10 & $25 \%$ & 18 & $36 \%$ \\
\hline
\end{tabular}

Fuente: Elaboración propia. 
Gobierno corporativo y ReEmplazo de Presidentes de

los Consejos de Administración en MéXico

el Presidente de la Junta Directiva de una empresa tiene en un año en particular, algún apellido igual a otro miembro del mismo Consejo de Administración. Se considera que una firma tiene vínculo familiar en la pre-crisis, crisis y/o post-crisis si posee este nexo en al menos uno de los años del período en cuestión. Se tiene vínculo empresarial cuando el Presidente de una Junta Directiva está participando en el mismo año como miembro de otra u otras Juntas Directivas. Se cataloga a una empresa con vínculo empresarial en la pre-crisis, crisis y/o post-crisis si tiene este vínculo en al menos uno de los años del período en cuestión. Cuando el Presidente de la Junta de Directores de una compañía ha sido Gobernador de algún Estado, Diputado Federal electo mediante votación popular, Senador de la República o Secretario de Estado (de 1990 a 2000), se dice que esa empresa posee vínculo político. Se contempla que una firma tiene vínculo político en la pre-crisis, crisis y/o post-crisis si posee este nexo en al menos uno de los años del período en cuestión. Se considera una empresa con Junta de Directores Grande cuando el número de miembros es de 19 o más. Si la empresa en particular tiene Junta Directiva Grande en al menos 60\% de los años del período en estudio (pre-crisis, crisis o post-crisis), entonces se contabiliza como una empresa con Junta Directiva Grande durante ese tiempo.

Observando esta tabla en cada uno de los sub-períodos de estudio, no existe una norma de comportamiento clara, sin embargo, tanto en la crisis como en la post-crisis, el mayor número de reemplazos se dio en aquellas firmas que no poseen ninguna de las estrategias de Gobierno Corporativo contempladas.

\subsection{DESEMPEÑO PREVIO, DURANTE Y POSTERIOR A LOS REEMPLAZOS}

En la Tabla 3 se muestra el rendimientos sobre los activos (ROA) promedio que tuvieron las firmas mexicanas en situaciones previas, durante y posteriores a los reemplazos de los Presidentes de las Juntas Directivas, de acuerdo a las estrategias de Gobierno Corporativo adoptadas.

En esta tabla, las 16 características (excluyendo la categoría "todas las firmas") que se muestran son mutuamente excluyentes y exhaustivas. Para cada empresa, el ROA previo al reemplazo del Presidente de la Junta Directiva se refiere al 
observado el año anterior al mismo; el ROA durante el reemplazo es aquel propiamente en el año en que se da este evento; el ROA posterior al reemplazo se refiere al observado un año luego del cambio de este funcionario. Para calcular el ROA promedio (promedio simple) previo, durante y posterior al reemplazo de los Presidentes de las Juntas Directivas de las empresas en cuestión, primero se eliminan los 42 valores extremos (ROA mayor a $130 \%$ y menor a $-80 \%$, en el período de 1990 a 2000). Entre paréntesis se encuentra la desviación estándar. El vínculo familiar se establece cuando el Presidente de la Junta Directiva de una empresa tiene, en un año en particular, algún apellido igual a otro miembro del mismo Consejo de Administración. Se tiene vínculo empresarial cuando el Presidente de una Junta Directiva está participando en el mismo año como miembro de otra u otras Juntas Directivas. Cuando el Presidente de la Junta de Directores de una compañía ha sido Gobernador de algún Estado, Diputado Federal electo mediante votación popular, Senador de la República o Secretario de Estado (de 1990 a 2000), se dice que esa empresa posee vínculo político. Se considera una empresa con Junta de Directores Grande cuando el número de miembros para un año en particular es de 19 o más.

Se observa que, en muchos de los casos, el rendimiento previo al reemplazo fue negativo. Esto indica que el cambio en el Presidente de la Junta Directiva pudo darse por mal desempeño. No obstante, en general el cambio en el Presidente de la Junta Directiva no favoreció al desempeño empresarial ni en el corto plazo (durante el año del reemplazo) ni en el mediano plazo (un año posterior al cambio). Algunas excepciones notables de esto se dieron en aquellas empresas con desempeño muy pobre previo al reemplazo.

\section{RESULTADOS ECONOMÉTRICOS}

\subsection{MODELO ECONOMÉTRICO}

Para verificar las hipótesis planteadas se corren modelos Logit con el método de Huber/White. Como se explica en la metodología, la función estimada se expresa de la siguiente manera: 
Gobierno corporativo y ReEmplazo de Presidentes de

los Consejos de Administración en México

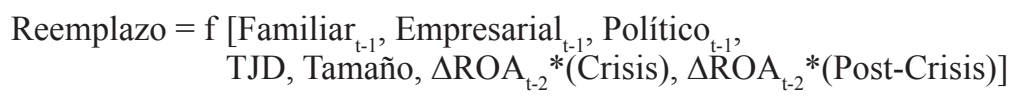

La función (1) se amplía para contemplar también el efecto de las cinco estrategias dominantes de Gobierno Corporativo (de acuerdo a la estadística descriptiva), sobre la probabilidad de reemplazo del Presidente del Consejo de Administración:

$$
\begin{aligned}
\text { Reemplazo }=\mathrm{f} & {\left[\mathrm{NGC}_{\mathrm{t}-1}, \mathrm{FEG}_{\mathrm{t}-1}, \mathrm{FEPG}_{\mathrm{t}-1}, \text { Familiar }_{\mathrm{t}-1}, \text { Empresarial }_{\mathrm{t}-1},\right.} \\
& \text { Tamaño, } \left.\Delta \mathrm{ROA}_{\mathrm{t}-2} *(\text { Crisis }), \Delta \mathrm{ROA}_{\mathrm{t}-2} *(\text { Post-Crisis })\right]
\end{aligned}
$$

\subsection{RESULTADOS}

En la primera columna de la Tabla A1 del apéndice se muestran los coeficientes obtenidos para la función (1). En la segunda y tercera columna de la misma tabla se presentan los errores estándar y los valores $z$, con los que se estipulan los grados de significancia de cada uno de los coeficientes. Tal como se puede observar, sólo las variables tamaño de firma y $\Delta \mathrm{ROA}_{\mathrm{t}-2}$ en el período de post-crisis son significativas (al 1\% y $10 \%$, respectivamente). Los signos negativos en ambos casos implican que, a mayor tamaño de la empresa menor es la probabilidad de reemplazo del Presidente de la Junta Directiva y, entre mayor sea el desempeño pasado menor es la probabilidad de reemplazo en la post-crisis. Ambos hallazgos se corroboran con la Tabla A2, que se refiere a los resultados de la función 2, y con la literatura: Según Lausten (2002), entre más grande sea una firma, más compleja es su gestión y, por ende, es más baja la probabilidad de que se sustituya a un alto funcionario. Por otro lado, autores tales como Kaplan (1994); Coffee (1999) y Huson et al. (2004) presentan evidencia de que los directivos son forzados a dejar las firmas cuando hay una larga y significativa caída en los resultados empresariales. De acuerdo a Chang y Shin (2006) y Heaney et al. (2007), esto es más evidente en las post-crisis, debido al minucioso monitoreo y control implementados en las empresas como consecuencia de estos episodios.

No obstante, de las tablas A1 y A2 se deduce también que las variables y estrategias de Gobierno Corporativo estudiadas no tienen ningún impacto sobre la 
probabilidad de reemplazo de los Presidentes de las Juntas Directivas en México, lo cual no es consistente con la literatura para otros países. Esto hace parecer que la alta incidencia de dueños en las Juntas Directivas de las firmas mexicanas (ya que aproximadamente el 70\% de las empresas en México son familiares; ver Dávila y Watkins, 2008) tiene mayor preponderancia en las decisiones de reemplazo que cualquiera de los factores estudiados en la presente investigación. De hecho, se observa gran estabilidad en estos puestos, lo cual favorece el argumento anterior; el $52 \%$ de las empresas de la muestra no presentaron reemplazos durante el período de estudio 1990-2000.

Un ejemplo de lo antes expuesto es que, en Cementos Mexicanos, S.A. de C.V. (CEMEX por su clave en la BMV) Lorenzo Zambrano funge como Presidente del Consejo de Administración así como Director General (CEO por sus siglas en inglés de Chief Executive Officer) y socio mayoritario con el 51\% de las acciones comunes de la compañía. Además de ser una empresa con vínculos familiares $^{8}$ muy claros, se presentan los vínculos empresariales muy acentuados, ya que el mismo Lorenzo Zambrano está presente en varios consejos de administración como lo son ICA, FEMSA, TLEVISA, VITRO y ALFA, ${ }^{9}$ entre otros.

Ese mismo caso se da con Teléfonos de México, S.A. de C.V. (TELMEX por su clave en la BMV) en donde Carlos Slim es el Presidente de la Junta de Directores y a su vez es el socio mayoritario con más del 51\% de las acciones comunes de la empresa. Es también una compañía con vínculos tanto familiares como empresariales ya que, al igual que en CEMEX, hijos y hermanos del socio mayoritario tienen posiciones dentro de la misma empresa así como en Juntas de Directores de otras firmas.

Estos dos ejemplos (que corresponden a dos de las empresas más grandes del país) se pueden extender para otras firmas, tales como BIMBO, ELEKTRA, TLEVISA y TVAZTCA, entre otras, donde los socios mayoritarios son, además,

${ }^{8}$ Varios de los hermanos e hijos de los dueños aparecen como miembros, tanto de las Juntas de Directores como de los cargos ejecutivos de la misma firma.

9 Se muestran los nombres de las claves que tienen estas empresas en la Bolsa Mexicana de Valores (BMV) para una mejor identificación y referencia de las mismas. 
Gobierno corporativo y ReEmplazo de Presidentes de

los Consejos de Administración en México

Presidentes de las Juntas Directivas. Esto hace que los resultados econométricos para el modelo planteado no tengan significancia estadística, ya que la "realidad" mexicana es diferente a la observada en la literatura para la mayoría de los países (dos excepciones son Italia y Alemania).

Dada la incongruencia entre la estructura empresarial mexicana y la identificada en la literatura para otros países, las relaciones entre tamaño de la firma y desempeño pasado en la post-crisis, con la probabilidad de reemplazo, deberían darse por factores distintos a los considerados. Específicamente podrían explicarse por la misma estructura empresarial donde, evidentemente los dueños, que son además Presidentes de las Juntas Directivas, no son reemplazados. Para verificar esto primero se manejó la base de datos que busca determinar tres rangos de empresas, de acuerdo al valor de sus activos totales: las pequeñas (0-3,5 millones de pesos, 92 empresas), las medianas (3,5-11,5 millones de pesos, 40 firmas) y las grandes (más de 11,5 millones de pesos, 39 compañías), encontrándose que, efectivamente, las empresas grandes tienen una mayor composición de dueños que podrían ser también Presidentes de las Juntas de Directores (ya que alrededor del 85\% del total de estas firmas son familiares). En cambio, esta característica se presenta en el $68 \%$ de las empresas medianas y el $59 \%$ de las pequeñas.

En cuanto al desempeño pasado y su repercusión significativa sobre la probabilidad de reemplazo del Presidente del Consejo de Administración se observa que, en la mayoría de los casos (52\%) estos reemplazos se dan dentro de las mismas familias que controlan a una firma. Lo anterior puede interpretarse como un castigo por mala gestión sin que se afecte la estructura familiar de la empresa. Por otro lado, el 48\% de las veces el reemplazo se debió a una recomposición en el control de la empresa, donde la familia que controlaba a la firma deja de ser su dueña.

\section{CONCLUSIONES}

La conclusión principal de este estudio es, que las variables de Gobierno Corporativo analizadas no tienen impacto sobre la probabilidad de reemplazo de los 
Presidentes de las Juntas Directivas de las empresas mexicanas. Este hallazgo no es consistente con la literatura para otros países, lo que sugiere que México es un caso especial. El 70\% de las empresas en México son familiares; la alta incidencia de dueños en las Juntas Directivas de estas firmas tiene mayor preponderancia en las decisiones de reemplazo que cualquiera de los factores estudiados en la presente investigación. Esta estructura empresarial favorece la estabilidad en los puestos de los Presidentes de las Juntas Directivas; de hecho, la mayoría de las compañías estudiadas no presentaron reemplazos durante el período de estudio (1990-2000) y el número total de reemplazos fue de tan sólo 121. Al analizar cada sub-período de tiempo se observa que, este número va aumentando aproximadamente en un $30 \%$, pasando de 30 reemplazos en la pre-crisis a 40 durante la crisis y, finalmente, 51 en la post-crisis. No es de esperar un aumento en los cambios de Presidentes de las Juntas Directivas en tiempos de crisis, pero sí en la post-crisis donde, como reacción ante la crisis, hay mayor monitoreo y control, y posiblemente desgaste de quienes ocuparon cargos altos en la organización durante este episodio. En general se aprecia que, posterior a los reemplazos cae el desempeño empresarial, al menos en el corto plazo.

Otro resultado interesante es que a mayor tamaño de la firma, menor la probabilidad de reemplazo del Presidente de la Junta Directiva. Esta conclusión se observa también en la literatura para otros países, donde se argumenta que la complejidad de labores de las grandes corporaciones favorece la estabilidad de estos cargos. Sin embargo, para México la explicación es otra: prácticamente el 85\% de las empresas más grandes son familiares (y por ende los reemplazos son casi inexistentes); este porcentaje es del 68\% para las empresas medianas y de tan sólo $59 \%$ para las firmas pequeñas.

Por último, en cuanto al desempeño pasado y su repercusión sobre la probabilidad de reemplazo del Presidente del Consejo de Administración se observa que, en la mayoría de los casos estos reemplazos se dan dentro de las mismas familias que controlan a una firma. Lo anterior puede interpretarse como un castigo por mala gestión, sin que se afecte la estructura familiar de la empresa mexicana. En el resto de las veces el reemplazo se debió a una recomposición en el control de la empresa, donde la familia que controlaba a la firma deja de ser su dueña. 
Gobierno corporativo y ReEmplazo de Presidentes de

los Consejos de Administración en México

\section{BIBLIOGRAFÍA}

Aggarwal, Rajesh K. y Andrew A. Samwick (1999), "Executive compensation, strategic competition, and relative performance evaluation: Theory and evidence", Journal of Finance 54, 1999-2043.

Agrawal, Anup y Charles R. Knoeber (1996), "Firm performance and mechanisms to control agency problems between managers and shareholders", Journal of Financial and Quantitative Analysis 31, 377-397.

Agrawal, Anup y Charles R. Knoeber (2001), "Do some outsider directors play a political role?", Journal of Law and Economics 44, 179-199.

Alchian, W.A. y H. Demsetz (1972), "Production, information costs, and economic organization", American Economic Review, 62, 777-795.

Anderson, R.C. y D.M. Reeb (2003), "Founding-family ownership and firm performance: Evidence from the S\&P 500”, Journal of Finance 58, 13011328.

Barro, J. y R. Barro (1990), "Pay, performance and turnover of Bank CEOs", Journal of Labor Economics 8, 448-481.

Bebchuk, Lucian A. y Jesse M. Fried (2003), "Executive Compensation as an Agency Problem", Journal of Economic Perspectives 3, 71-92.

Bebchuk, Lucian A., A. Cohen y A. Ferrell (2004), "What matters in corporate governance?”, Harvard University Law School working papers.

Bebchuk, Lucian A., Martijn Cremers y Urs Peyer (2008), “CEO Centrality”, Harvard University working papers.

Bebchuk, Lucian A., Jesse M. Fried y David I. Walker (2002), "Managerial Power and Rent Extraction in the Design of Executive Compensation", University of Chicago Law Review 69.

Bennedsen, Morten, Kasper M. Nielsen, Daniel Wolfenzon y Francisco PérezGonzález (2007), "Inside the Family Firm: The Role of Families in Succession Decisions and Performance", The Quarterly Journal of Economics.

Bhagat, Sanjai y Bernard S. Black (2002), "The non-correlation between board independence and long term performance", Journal of Corporation Law 27, 231-274. 
Bhagat, Sanjai y Brian Bolton (2008), "Corporate governance and firm performance”, Journal of Corporate Finance, 118 (1), 107-155.

Bonnier, K. A. y R.F. Bruner (1989), "An analysis of stock price reaction to management change in distressed firms", Journal of Accounting and Economics $11,95-106$.

Chang, James J. y Hyun-Han Shin (2006), Governance System Effectiveness Following the Crisis: the case of Korean business group headquarters, Corporate Governance 14, 85-97.

Claessens, Stijn.; Simeon Djankov; Joseph P.H. Fan y Larry H.P. Lang (2002), "Disentangling the incentive and entrenchment effects of large shareholdings", Journal of Finance 57, 2741-2772.

Coffee, J. (1999), "The Future as history: the prospects for global convergence in corporate governance and its implications", Northwestern University Law Review 93, 641-708.

Conyon, Martin J. (1998), "Directors' pay and Turnover: An application to a Sample of Large UK Firms", Oxford Bulletin of Economics and Statistics 60, 485-507.

Conyon, Martin J. y Annita Florou (2002), “Top Executive Dismissal, Ownership and Corporate Performance", Accounting and Business Research 32, 209-226.

Cosh, A. y A. Hughes (1997), "Executive remuneration, executive dismissal and institutional shareholdings", International Journal of Industrial Organization 15, 469-492.

Coughlan, Anne T. y Ronald M. Schmidt (1985), "Executive compensation, management turnover and firm performance: an empirical investigation", Journal of Accounting and Economics 7, 43-66.

Dahya, J.; A. Lonie y D. Power (1998), "Ownership structure, firm performance, and top executive change: An analysis of UK firms", Journal of Business Finance and Accounting 25, 1089-1118.

Dávila, Martín y Karen Watkins (2008), Corporate Governance and Firm Performance Lessons from de Mexican Crisis (1994-1995), Making Capital Markets Work Through Corporate Governance, ed. World Council for Corporate Governance, London 2008, 45-73. 
Gobierno corporativo y ReEmplazo de Presidentes de

los Consejos de Administración en México

DeFond, Mark L. y C.W. Park (1999), "The effect of competition en CEO turnover", Journal of Accounting and Economics 27, 35-56.

Denis, D.J. y Diane K. Denis (1995), "Performance changes following top management dismissals", Journal of Finance 50, 1029-1057.

Denis, David J.; Diane K. Denis y Atulya Sarin (1997), “Ownership structure and top executive turnover", Journal of Financial Economics 52, 187-223.

Dyck, Alexander y Luigi Zingales (2004), "Private benefits of control: an international comparison", Journal of Finance 59, 537-600.

Eisenberg, Theodore; Stefan Sundgren y Martin T. Wells (1998) "Larger board size and decreasing and increasing firm solvency", Journal of Financial Economics 48, 35-54.

Faccio, Mara (2006a), "Politically connected firms", American Economic Review 96, 369-386.

Faccio, Mara; Ronald W. Masulis y John J. McConnell (2006b), "Political Connections and Corporate Bailouts", Journal of Finance 61, 2597-2635.

Fama, Eugene F. (1980), "Agency Problems and the Theory of the Firm”, Journal of Political Economy 88, 288-307.

Fama, Eugene F. y Michael C. Jensen (1983a), "Separation of ownership and control", Journal of Law and Economics 26, 301-325.

Fama, Eugene F. y Michael C. Jensen (1983b), “Agency problems and residual claims", Journal of Law and Economics 26, 327-349.

Fan, Dennis K.K, Chung-Ming Lau y Michael Young (2007) “Is China's corporate governance beginning to come of age? The case of CEO turnover", PacificBasin Finance Journal, 15, 105-120.

Fan, Joseph P. H., T.J. Wong y Tianyu Zhang (2008), "Politically connected CEOs, corporate governance, and Post-IPO performance of China's newly partially privatized firms", Journal of Financial Economics, 84, 330-357.

Fee, C. Edward y Charles J. Hadlock (2000), "Management Turnover and product market competition: empirical evidence from the U.S. newspaper industry", Journal of Business 73, 205-243.

Fee, C. Edward y Charles J. Hadlock (2004), "Management Turnover across the Corporate Hierarchy", Journal of Accounting and Economics 37, 3-38. 
Ferris, Stephen P.; Murali Jagannathan y A.C. Pritchard (2003), "Too busy to mind the business? Monitoring by directors with multiple board appointments", Journal of Finance 58, 1087-1111.

Fich, Eliezar M. y Anil Shivdasani (2006), "Are Busy Boards Effective Monitors?", The Journal of Finance 51, 689-724.

Firth, Michael, Peter M. Y. Fung y Oliver M. Rui (2006), "Firm Performance, Governance Structure, and Top Management Turnover in a Transitional Economy", Journal of Management Studies 43, 1289-1330.

Fisman, Raymond (2001), "Estimating the value of political connections", American Economic Review 91, 1095-1102.

Florou, Anita (2005), “Top Director Shake-up: The link between Chairman and CEO Dismissal in the UK", Journal of Business Finance \& Accounting 43, 97-128.

Fung, Peter M.Y. y Oliver M. Rui (2006), "Firm Performance, Governance Structure, and Top Management Turnover in a Transitional Economy", Journal of Management Studies 43, 1289-1330.

Gibbons, R. y K.J. Murphy (1990), "Relative performance evaluation for Chief Executive Officers", Industrial and Labor Relations Review 43, 30S-51S.

Gibson, Michael S. (2003), "Is Corporate Governance Ineffective in Emerging Markets?", Journal of Financial and Quantitative Analysis 38, 231-250.

Gompers, P.A. J.L. Ishii y A. Metrick (2003), "Corporate governance and equity prices", Quaterly Journal of economicsa, 118 (1), 107-155.

Harrison, J.R.; D.L. Torres y S. Kukalis (1988), "The changing of the guard: turnover and structural change in the top-management positions", Administrative Science Quarterly 33, 211-232.

Heaney, Richard, Tony Naughton, Thanh Truong, Sinclair Davidson, Tim Fry y Michael McKenzie (2007), "The link between performance and changes in the size and stability of a firm's officers and directors", Journal of Multinational Financial Management, 17, 16-29.

Hermalin, Benjamin E. y Michael S. Weisbach (1998), "Endogenously chosen Boards of directors and their monitoring of the CEO", The American Economic Review 88, 96-118. 
Gobierno corporativo y ReEmplazo de Presidentes de

los Consejos de Administración en México

Hillier, David J. y Patrick M.L. McColgan (2004), "Firm Performance, Entrenchment and managerial Succession in Family Firms", University of Aberdeen working paper.

Hillier, David, Scott C. Linn y Patrick McColgan (2005), "Equity Issuance, CEO Turnover and Corporate Governance", European Financial Management 11, 515-538.

Hoskisson, R.E., M. Hitt, W. Wan y D. Yiu (1999), "Theory and research, in strategic management: swings of a pendulum", Journal of Management 25, 417-456.

Huson, Mark R.; Paul H. Malatesta y Robert Parrino (2004), "Managerial succession and firm performance", Journal of Financial Economics 74, 237-275.

Jensen, Michael C. (1993), "The Modern Industrial Revolution, Exit, and the failure of internal control systems", Journal of Finance 48, 831-880.

Jensen, Michael C. y K.J. Murphy (1990), "Performance pay and top management incentives", Journal of Political Economy 98, 225-264.

Jenter, Dirk C. y Fadi Kanaan (2006), "CEO Turnover and Relative Performance Evaluation", MIT Sloan Research Paper, no. 4594-06.

Jovanovic, Boyan (1979), "Job Matching and the Theory of Turnover", Journal of Political Economy 87, 972-990.

Kaplan, Steven N. (1994), "Top Executives, Turnover, and Firm Performance in Germany", Journal of Law Economics and Organization 10, 142-159.

Khanna, Naveen y Annette B. Poulsen (1995), "Managers of financially distressed firms: villains or scapegoats?", Journal of Finance 50, 919-940.

La Porta, Rafael, Florencio López-de-Silanes, Andrei Shleifer y Robert W. Vishny (1998), "Law and finance", Journal of Political Economy 106, 1115-1155.

Lausten, Mette (2002), "CEO turnover, firm performance and corporate governance: empirical evidence on Danish firms”, International Journal of Industrial Organization 20, 391-414.

Lipton, M. y Jay W. Lorsch (1992), "A modest proposal for improved corporate governance", Business Lawyer 59, 59-77.

Mak Y.T. y Yuan Li (2001), "Determinants of corporate ownership and board structure: evidence from Singapore", Journal of Corporate Finance 7, 235-256. 
Martín DÁvila / Karen WatKins

Manne, Henry G. (1965), "Mergers and the Market for Corporate Control", Journal of Political Economy, 110-120.

Maury, Benjamin (2006), "Corporate Performance, Corporate Governance and Top Executive Turnover in Finland", European Financial Management 12, 221-248.

McKenna, C. J. (1986), "Equilibrium Wage Offers and Turnover in a Simple Search Market", The Economic Journal 96, 785-797.

McNeil, Chris; Greg Niehaus y Eric Powers (2004), "Management Turnover in Subsidiaries of Conglomerates Versus Stand-alone Firms", Journal of Financial Economics 72, 63-96.

Murphy, Kevin J. (1986), "Incentives, Learning and Compensation: A Theoretical and Empirical Investigation of Managerial Labor Contracts", Rand Journal of Economics 17, 59-76.

Nair, Vinay B. (2005), “Corporate Governance and Internal Organization”, Wharton School at University of Pennsylvania working paper.

Nenova, Tatiana (2000), "The value of corporate votes and control benefits: a cross-country analysis", Harvard University working paper.

Parrino, Robert (1997), "CEO turnover and outside succession: a cross-sectional analysis", Journal of Financial Economics 46, 165-197.

Perry, Tod (1999), "Incentive Compensation for Outside Directors and CEO Turnover", Arizona State University working paper.

Roberts, Brian E. (1990), "A dead senator tells no lies: Seniority and the distribution of federal benefits", American Journal of Political Science 34, 31-58.

Stulz, René M. (1988), "Managerial control of voting rights: financing policies and the market for corporate control", Journal of Financial Economics 20, 25-54.

Sung, Q. y W. Tong (2003), "China share issue privatization: the extent of its success", Journal of Financial Economics 70, 183-222.

Tosi, Henry L., Wei Shen y Richard J. Gentry (2003), "Why Outsiders on Boards Can’t Solve the Corporate Governance Problem”, Organizational Dynamics 32, 180-192.

Villalonga, Belén y Raphael Amit (2006), "How do family ownership, control, and management affect firm value?”, Journal of Financial Economics 80, 385-417. 
Volpin, Paolo F. (2002), "Governance with poor investor protection: evidence from top executive turnover in Italy", Journal of Financial Economics 64, 61-90.

Warner, Jerold B.; Ross L. Watts y Karen H. Wruck (1988), "Stock prices and top management changes", Journal of Financial Economics 20, 461-492.

Weisbach, Michael S. (1988), "Outside directors and CEO Turnover", Journal of Financial Economics 20, 431-460.

Yermack, David (1996), "Higher market valuation of companies with a small board of directors", Journal of Financial Economics 40, 185-211. 


\section{APÉNDICE}

TABla A1

Probabilidad de reemplazo de Presidentes de las Juntas Directivas en México, durante el período de estudio 1990-2000, de acuerdo a variables de Gobierno Corporativo

$\begin{array}{lccc}\text { Variable } & \text { Coeficiente } & \text { Error estándar } & \text { Estadístico Z } \\ \text { Vínculo familiar (-1) } & & & \\ \text { Vínculo empresarial(-1) } & -0.28 & 0.41 & -0.69 \\ \text { Vínculo político (-1) } & 0.41 & 0.32 & 1.28 \\ \text { Junta Directiva Grande } & 0.50 & 0.66 & 0.76 \\ \text { Tamaño de la firma } & -0.04 & 0.76 & -0.05 \\ \text { Cambio en ROA (-2) crisis } & -0.14^{* *} & 0.01 & -15.96 \\ \text { Cambio en ROA (-2) post-crisis } & -1.88 & 2.19 & -0.86 \\ \end{array}$

$*, * *$, niveles de significancia al $10 \%$ y $1 \%$, respectivamente.

En la Tabla A1 se presentan los resultados de las estimaciones del modelo econométrico (1), empleando una estimación Logit.

\section{TABLA A2}

Probabilidad de reemplazo de Presidentes de las Juntas Directivas en México, durante el período de estudio 1990-2000, de acuerdo a las estrategias dominantes de Gobierno Corporativo

$\begin{array}{llcc} & \text { Coeficiente } & \text { Error estándar } & \text { Estadístico Z } \\ \text { Variable } & & & \\ & & & -1.12 \\ \text { Vínculo familiar (-1) } & -0.62 & 0.55 & 0.18 \\ \text { Vínculo empresarial (-1) } & 0.08 & 0.47 & 0.44 \\ \text { Vínculo familiar, empresarial y Junta } & 0.21 & 0.47 & -0.83 \\ \text { Directiva Grande (-1) } & & & -1.65 \\ \text { Vínculo familiar, empresarial, político } & -0.43 & 0.52 & -4.25 \\ \text { y Junta Directiva Grande (-1) } & -0.67^{*} & 0.41 & -0.85 \\ \text { Sin ninguna estrategia } & -0.11^{* *} & 0.03 & -1.66 \\ \text { Tamaño de la firma } & -1.92 & 2.25 & \\ \text { Cambio en ROA (-2) crisis } & -1.16^{*} & 0.70 & \\ \text { Cambio en ROA (-2) post-crisis } & & & \end{array}$

$*$,**, niveles de significancia al $10 \%$ y $1 \%$, respectivamente.

En la Tabla A2 se presentan los resultados de las estimaciones del modelo econométrico (2), empleando una estimación Logit. 